\title{
Motivos de permanência dos praticantes nos programas de exercícios físicos oferecidos pelo Serviço Social do Comércio - Brasil
}

\author{
Practitioners' reasons for remaining in physical exercise programs offered \\ by the Social Service of Commerce - Brazil
}

\author{
M.S. Castro, N.L. Silva, W. Monteiro, A. Palma, H.G. Resende
}

\begin{abstract}
Uma das preocupações nos estudos de adesão recai na investigação dos motivos que justificam a permanência dos praticantes em programas de exercícios físicos. Neste sentido, o objetivo da presente investigação foi identificar os grupos (categorias) de motivos que influenciam a permanência de adolescentes, adultos e idosos nos programas de exercícios físicos oferecidos nos Centros de Atividades do Serviço Social do Comércio do Distrito Federal do Brasil. Uma amostra representativa e aleatória de 986 indivíduos de ambos os sexos (15-89 anos) respondeu a um instrumento de coleta de dados para investigar aspectos sócio-demográficos e os motivos de permanência no referido programa. Os resultados revelaram que o bem-estar pessoal (91.9\%) foi considerado o principal motivo para a manutenção da prática de exercícios físicos, entre todas as possibilidades existentes, seguido pela prevenção de doenças $(82.8 \%)$, condicionamento físico $(80.7 \%)$, empatia com o professor $(79.2 \%)$, metodologia do trabalho desenvolvido $(75.2 \%)$ e diversão $(73.9 \%)$. Enquanto a prescrição médica tem alto potencial para estimular a inserção destes praticantes nos referidos programas, este motivo perde força no sentido de justificar a permanência da prática de exercícios físicos. Deste modo, é possível concluir que o bem-estar pessoal foi considerado o principal motivo alegado pelos participantes dos programas de exercícios físicos oferecidos pelo Serviço Social do Comércio - Brasil.
\end{abstract}

Palavras-chave: exercício físico, adesão, fatores motivacionais

ABSTRACT

One of the concerns in the studies of adhesion lies in the investigation of the motives that justify continuation in physical exercise programs. The goal of this study was to identify motives influencing the continuation of adolescents, adults and the elderly in physical education programs offered by the Centers of Activity of Serviço Social do Comércio do Distrito Federal do Brasil. A representative and random sample of 986 individuals (between 15 and 89 years of age) answered a data collection instrument designed to investigate socio-demographic aspects and motives for permanence in the aforementioned program. Results showed personal wellbeing $(91.9 \%)$ as the major reason for keeping up on practicing followed by prevention of illnesses $(82.8 \%)$, physical conditioning (80.7\%), empathy with the teacher $(79.2 \%)$, program methodology $(75.2 \%)$, and entertainment $(73.9 \%)$. While medical exercise prescription highly influences people's entrance in these programs, it does not explain people's continuance.

Keywords: physical exercise, adherence, motivating factors

Submetido: 29.09.2009 | Aceite: 12.05.2010

\footnotetext{
Mônica da Silva Castro. Serviço Social do Comércio - Brasil.

Nádia Lima da Silva. Universidade do Estado do Rio de Janeiro - Brasil.

Walace Monteiro. Universidade do Estado do Rio de Janeiro e Universidade Salgado de Oliveira - Brasil.

Alexandre Palma. Universidade Federal do Rio de Janeiro - Brasil.

Helder Guerra de Resende. Universidade Federal de Juiz de Fora e Universidade Castelo Branco - Brasil.

Endereço para correspondência: Helder Guerra de Resende, Rua Geminiano Góis, 1300 - Apto. 1302, CEP 22.743-

670, Rio de Janeiro - RJ - Brasil.

E-mail: heldergr@globo.com
} 
A literatura científica tem reforçado a crença sobre os benefícios da prática regular e adequada de exercícios físicos para prevenção de doenças. Diversos estudos têm tentado demonstrar que esta conduta está associada à prevenção de doenças cardiovasculares, diabetes, obesidade, osteoporose e depressão (Haskell et al., 2007; U. S. Department of Health and Human Services, 1996; Waxman, 2004), muito embora a noção de causalidade entre exercício físico e saúde esteja sendo questionada por diferentes pesquisadores (Mira, 2003; Williams, 2003).

Em investigação desenvolvida no Serviço Social do Comércio - SESC (2003) demonstrou-se que a população tem conhecimento e acredita nos benefícios da realização de exercícios físicos para prevenção de doenças e, no entanto, contraditoriamente, não tem aderido à prática regular deste comportamento (CDC, 2000; Fechio \& Malerbi, 2004; Ferreira \& Najar, 2005; Souza \& Duarte, 2005). Essa afirmação está apoiada no entendimento de que a literatura considera que há baixas taxas de engajamento de pessoas em programas regulares de exercícios físicos (Hallal, Bertoldi, Gonçalves, \& Victora, 2006; Monteiro, Conde, Matsudo, Matsudo, Bonseñor, \& Lotufo, 2003; Varo, MartínezGonzález, Irala-Estévez, Kearney, Gibney, \& Martínez, 2003), a despeito destas medidas serem, muitas vezes, questionadas (Pate, O'Neill, \& Lobelo, 2008; Williams, 2003).

$\mathrm{Na}$ medida em que a prática regular de exercícios físicos tem sido considerada importante para a prevenção de diferentes doenças crônico-degenerativas e para melhoria da aptidão física, tem sido observado que o estímulo à prática parece estar focado na perspectiva da saúde como um fim em si mesmo. Por outro lado, é interessante notar que alguns autores têm demonstrado que indivíduos aparentemente sem doenças não têm investido na prática de exercícios físicos com o objetivo específico de prevenção primária (Alves, Junger, Palma, Monteiro, \& Resende, 2007; Castro, Miranda, Silva, Palma,
\& Resende, 2009; Lovisolo, 2000). Diante de uma doença que ainda não se manifestou, e talvez nunca se manifeste, a hipótese é que as pessoas que apresentem um perfil sadio devem aderir a um programa de exercícios físicos a partir de razões variadas, tais como os interesses ligados ao gosto, ao prazer, à melhoria do condicionamento físico e/ou da estética corporal, entre outros.

Em resposta a essa questão, tem-se verificado um aumento gradativo de investigações voltadas para a denúncia acerca da limitação da concepção biomédica de saúde, nas quais se considera que os contextos sócioeconômico, ambiental e histórico-cultural de indivíduos e populações poderiam explicar melhor o fenômeno da adesão à prática regular de exercícios físicos (Castro et al., 2009; Ferreira \& Najar, 2005; Palma, Ferreira, Bagrichevsky, \& Resende, 2006; Williams, 2003).

Diante do quadro apresentado, estudos têm sido desenvolvidos com o objetivo de entender a problemática da adesão dos indivíduos à prática de exercícios físicos. No entanto, a grande maioria dos estudos demonstra preocupação com os motivos que levaram o indivíduo a ingressar em programas dessa natureza (Alves et al., 2007; Andreotti \& Okuma, 2003; Castro et al., 2009; Vieira \& Ferreira, 2004). Para Andreotti e Okuma (2003), os motivos que levam ao início da prática de exercícios físicos podem não ser os mesmos que levam os indivíduos a permanecerem praticando-os.

De acordo com Dishman (1998), boa parte das pessoas que começam ou recomeçam um programa de exercícios físicos tendem a interromper com suas intenções de manutenção da prática. No entanto, a mesma atenção não tem sido dispensada aos aspectos que tratam da manutenção desses indivíduos nos referidos programas, em especial no nosso país (Alves et al., 2007).

Diante desse quadro, existe a necessidade de ampliar as investigações sobre o tema, principalmente considerando programas 
direcionados a grandes massas populacionais. Nessa direção, o presente estudo, tem por objetivo identificar os grupos (categorias) de motivos que influenciam a permanência de praticantes inscritos nos programas de exercícios físicos oferecidos nos Centros de Atividades do Serviço Social do Comércio localizados no Distrito Federal - Brasil (SESCDF).

O SESC é uma entidade privada de ação social, que promove programas educativos, prioritariamente voltados para o bem-estar de trabalhadores do comércio de bens e serviços, seus familiares e dependentes, como também, da comunidade em geral. A entidade é mantida pelos empresários do setor, oferecendo diversos programas nas áreas da educação, saúde, lazer, cultura e assistência, e está presente em todos estados brasileiros.

\section{Amostra}

\section{MÉTODO}

Os sujeitos foram selecionados a partir de um quantitativo de 12845 indivíduos, com idades compreendidas entre 15 e 89 anos, inscritos em pelo menos uma modalidade de exercício físico (ginástica ou esporte) dos seis Centros de Atividades do SESC-DF. Foram eliminadas por sorteio as replicações de alunos inscritos em mais de uma modalidade de exercício físico ou turma, de forma a manter a mesma probabilidade de seleção.

Tabela 1

Descrição da amostra por Centro de Atividades do SESC

\begin{tabular}{lcc}
\hline Unidade do SESC & População & Amostra \\
\hline 504 Sul & 3208 & 246 \\
913 Sul & 1895 & 145 \\
Gama & 1015 & 79 \\
Guará & 1762 & 135 \\
Taguatinga Norte & 3620 & 278 \\
Taguatinga Sul & 1345 & 103 \\
\hline Total & 12845 & 986 \\
\hline
\end{tabular}

A amostra foi composta de forma aleatória simples, sendo representativa do SESC-DF e proporcional a cada Centro de Atividades (Unidades do SESC-DF), totalizando 986 sujeitos de ambos os sexos, definidos por sorteio (ver tabela 1 ).

Antes da coleta de dados, os indivíduos foram informados acerca dos propósitos desta investigação e assinaram um termo de consentimento livre esclarecido. A pesquisa foi aprovada pelo Comitê de Ética Institucional.

\section{Instrumentos e Procedimentos}

Esta investigação seguiu as orientações técnicas do método da pesquisa do tipo survey interseccional (Babbie, 2001). Os dados foram coletados em um período de 10 dias, em cada Centro de Atividades do SESC-DF, através de um instrumento de coleta de dados autoadministrado, adaptado de uma versão originalmente desenvolvida e validada por Alves et al. (2007). O instrumento de coleta de dados foi constituído de duas partes, quais sejam: (a) um questionário sobre os aspectos sócio-demográficos dos praticantes de atividades físico-desportivas e (b) uma escala de opiniões sobre os motivos de permanência no programa de exercício físico. Nessa parte, as opiniões dos avaliados foram agrupadas por categorias, a saber: bem-estar pessoal, prevenção de doenças, condicionamento físico, empatia com o professor, metodologia de trabalho, diversão, ambiente físico, facilidade de horário e acesso ao local de prática, relacionamento social, estética corporal, custo, influência de terceiros, desafio, reconhecimento social, atividade terapêutica, rendimento esportivo (Alves et al., 2007). A necessidade de adequação do instrumento de coleta de dados deve-se ao fato da versão original ter sido desenvolvida para aplicação junto a um grupo de adolescentes praticantes de natação. Portanto, foram processadas reformulações nos indicadores específicos originalmente focados na natação, bem como, naqueles direcionados particularmente aos adolescentes, considerando que a presente investigação abrangeu faixas etárias variadas (adolescentes, adultos e idosos) e um universo mais amplo de modalidades de exercícios físicos (esportes e ginásticas em geral). 
O instrumento de coleta de dados foi aplicado pelos professores de Educação Física responsáveis pela prescrição e orientação das diferentes modalidades de exercícios físicos praticadas pelos indivíduos que constituíram a amostra. Apesar de auto-administrado, os indivíduos tiveram a oportunidade de esclarecer dúvidas com os referidos profissionais, que foram previamente treinados em dia, hora e local comum (um dos Centros de Atividades do SESC-DF) para a adequada aplicação e esclarecimentos sobre o instrumento de coleta de dados. Todos os instrumentos de coleta de dados foram preenchidos antes do início das sessões de exercícios físicos.

\section{Análise Estatística}

O tratamento dos dados foi realizado em duas etapas.

A primeira envolveu a verificação da confiabilidade da escala de opinião do instrumento de coleta de dados por meio do cálculo do coeficiente de consistência interna da escala (coeficiente alpha de Cronbach).

Em seguida, os dados foram tratados através de estatística descritiva, tomando-se como base a caracterização da amostra segundo o sexo, a faixa etária, a escolaridade, a renda familiar, as modalidades de exercícios físicos praticadas e a situação conjugal.

A fim de verificar quais os principais motivos que justificam a permanência dos usuários nos programas de exercícios físicos do SESC-DF, registraram-se os valores percentuais conferidos a cada categoria da escala, obedecendo a classificação registrada pelos respondentes para cada indicador: "muito importante", "mais ou menos importante" ou "nada importante". Para determinação dos principais motivos foram consideradas as categorias que obtiveram o percentual igual ou superior a $70 \%$, parâmetro de consenso de opiniões definido em função do critério definido por Bellack, Kliebard e Smith (1966).
Os dados foram analisados no programa SPSS v13.

\section{RESULTADOS}

A verificação da confiabilidade da escala de opinião do questionário mostrou um coeficiente alpha de Cronbach de .90, o que indica a existência de consistência interna entre as categorias do instrumento.

Quanto à caracterização sócio-demográfica, a tabela 2 apresenta uma síntese da amostra estudada, onde são descritos os itens que apresentaram maior prevalência e importância para a análise dos dados. Ressalta-se que os dados encontrados no tempo de permanência demonstram um bom índice de adesão dos participantes aos programas de exercícios físicos, totalizando $74.6 \%$ dos indivíduos com mais de seis meses de prática sem interrupção.

Tabela 2

Caracterização sócio-demográfica da amostra

\begin{tabular}{|c|c|}
\hline Variáveis & $\%$ \\
\hline \multicolumn{2}{|l|}{ Idade } \\
\hline $21-40$ anos & 44.6 \\
\hline $41-60$ anos & 28.2 \\
\hline Demais faixas etárias & 27.2 \\
\hline \multicolumn{2}{|l|}{ Sexo } \\
\hline Feminino & 64.9 \\
\hline Masculino & 35.1 \\
\hline \multicolumn{2}{|l|}{ Renda familiar } \\
\hline Acima de $\mathrm{R} \$ 3.000,00$ & 41.7 \\
\hline Entre $\mathrm{R} \$ 1.800,00$ e $\mathrm{R} \$ 3.000,00$ & 25.3 \\
\hline Abaixo de $\mathrm{R} \$ 1.800,00$ & 33.0 \\
\hline \multicolumn{2}{|l|}{ Escolaridade } \\
\hline Nível superior & 41.4 \\
\hline Demais níveis & 58.6 \\
\hline \multicolumn{2}{|l|}{ Situação conjugal } \\
\hline União estável & 51.9 \\
\hline Solteiros & 34.1 \\
\hline Demais situações & 14.0 \\
\hline \multicolumn{2}{|l|}{ Tipo de atividade } \\
\hline Ginástica & 57.9 \\
\hline Esportes individuais & 38.4 \\
\hline Demais modalidades & 3.7 \\
\hline \multicolumn{2}{|l|}{ Tempo de prática sem interrupção } \\
\hline Menos de 6 meses & 25.4 \\
\hline$>6$ meses a 1 ano & 24.3 \\
\hline$>1$ ano & 50.3 \\
\hline
\end{tabular}


A tabela 3 ilustra os resultados da escala de opinião, que indicam os motivos de permanência dos indivíduos na prática de exercícios físicos. As categorias que apontam interesses relacionados ao bem-estar pessoal, à prevenção de doenças, ao condicionamento físico, à empatia com o professor, à metodologia de trabalho desenvolvida pelo professor e à diversão foram as que atingiram maiores percentuais ( $>70 \%)$, sendo classificadas como os motivos mais importantes para a permanência, ilustrando razões vinculadas à utilidade e ao gosto.

Tabela 3

Distribuição percentual dos indivíduos da amostra de acordo com a escala de opinião referente ao nível de importância para permanência nos programas de exercícios físicos

\begin{tabular}{lcccc}
\hline \multicolumn{1}{c}{ Categorias } & \multicolumn{4}{c}{ Nível de importância } \\
\cline { 2 - 5 } & $\begin{array}{c}\text { Muita } \\
(\%)\end{array}$ & $\begin{array}{c}\text { Regular } \\
(\%)\end{array}$ & $\begin{array}{c}\text { Nenhuma } \\
(\%)\end{array}$ & $\begin{array}{c}\text { NR } \\
(\%)\end{array}$ \\
\hline Bem-estar pessoal & 91.9 & 6.6 & .9 & .6 \\
Prevenção de doenças & 82.8 & 13.7 & 2.5 & 1.0 \\
Condicionamento físico & 80.7 & 16.5 & 2.2 & .5 \\
Empatia com o professor & 79.2 & 16.1 & 4.0 & .6 \\
Metodologia de trabalho & 75.2 & 19.8 & 4.2 & .8 \\
Diversão & 73.9 & 19.4 & 6.1 & .6 \\
Ambiente físico & 58.8 & 33.1 & 7.2 & 1.0 \\
Facilidade de horário e acesso ao local da prática & 50.2 & 26.6 & 22.1 & 1.1 \\
Relacionamento social & 49.3 & 32.0 & 17.9 & .9 \\
Estética corporal & 48.2 & 34.7 & 16.7 & .5 \\
Custo & 47.2 & 24.3 & 27.6 & .9 \\
Influência de terceiros & 42.2 & 26.9 & 10.2 & .7 \\
Desafio & 40.7 & 36.3 & 22.2 & .9 \\
Reconhecimento social & 32.4 & 25.1 & 41.7 & .9 \\
Atividade terapêutica & 29.2 & 18.4 & 51.0 & 1.4 \\
Rendimento esportivo & 11.4 & 21.8 & 65.7 & 1.1 \\
\hline
\end{tabular}

NR - Não Respondeu

A tabela 4 apresenta as semelhanças e as diferenças encontradas para justificar a permanência dos indivíduos que praticam exercícios físicos, segundo a faixa etária. Nas faixas etárias de 15 a 20 anos, 21 a 40 anos e 41 a 60 anos de idade, as categorias consideradas mais importantes revelam-se idênticas às apontadas pelo total da amostra, variando apenas alguns valores percentuais. Os indivíduos com mais de 60 anos de idade, além das seis categorias comuns às outras faixas etárias, indicaram também o "ambiente físico" com $70.7 \%$ de registos.

Ainda na tabela 4 podem ser visualizadas as semelhanças e as diferenças encontradas para justificar a permanência dos indivíduos que praticam exercícios físicos, segundo o sexo. Tanto os indivíduos do sexo feminino quanto os do sexo masculino justificaram a permanência devido aos interesses relacionados ao bem-estar pessoal, à prevenção de doenças, ao condicionamento físico, à empatia com o professor, à metodologia de trabalho adotada pelo professor e à diversão. Quando considerado o corte de $70 \%$ adotado no presente estudo, a variável sexo não evidenciou diferenças em relação aos motivos que justificam a permanência em programas de exercícios físicos quando comparado com os resultados gerais da amostra. 
Tabela 4

Distribuição percentual dos indivíduos da amostra que reportaram muita importância para permanência nos programas de exercícios físicos, segundo a faixa etária e sexo

\begin{tabular}{|c|c|c|c|c|c|c|}
\hline \multirow[b]{2}{*}{ Categorias } & \multicolumn{4}{|c|}{ Faixa etária (anos) } & \multicolumn{2}{|c|}{ Sexo } \\
\hline & $\begin{array}{c}15-20 \\
(\%)\end{array}$ & $\begin{array}{c}21-40 \\
(\%)\end{array}$ & $\begin{array}{c}41-60 \\
(\%)\end{array}$ & $\begin{array}{l}>60 \\
(\%)\end{array}$ & $\begin{array}{c}\hat{O} \\
(\%)\end{array}$ & $\begin{array}{c}q \\
(\%) \\
(\%)\end{array}$ \\
\hline Bem-estar pessoal & 84.0 & 93.3 & 91.7 & 93.8 & 90.5 & 92.7 \\
\hline Prevenção de doenças & 74.1 & 81.1 & 84.7 & 89.5 & 77.5 & 85.6 \\
\hline Condicionamento físico & 80.7 & 81.8 & 78.7 & 85.6 & 80.3 & 81.0 \\
\hline Empatia com o professor & 79.2 & 74.5 & 83.5 & 92.2 & 74.2 & 82.0 \\
\hline Metodologia de trabalho & 75.2 & 72.6 & 80.0 & 83.0 & 70.0 & 78.1 \\
\hline Diversão & 73.9 & 71.6 & 74.9 & 85.8 & 70.3 & 75.8 \\
\hline Ambiente físico & 58.8 & 55.9 & 62.2 & 70.7 & 53.3 & 61.7 \\
\hline Facilidade de horário e acesso ao local da prática & 50.2 & 51.4 & 50.1 & 55.7 & 46.8 & 52.1 \\
\hline Relacionamento social & 49.3 & 42.4 & 53.1 & 65.8 & 45.8 & 51.2 \\
\hline Estética corporal & 48.2 & 51.6 & 42.2 & 47.1 & 47.6 & 48.5 \\
\hline Custo & 47.2 & 47.2 & 48.2 & 53.1 & 43.2 & 49.3 \\
\hline Influência de terceiros & 42.2 & 37.7 & 47.1 & 55.6 & 36.5 & 45.2 \\
\hline Desafio & 40.7 & 38.6 & 41.6 & 47.8 & 41.2 & 40.4 \\
\hline Reconhecimento social & 32.4 & 29.9 & 30.9 & 43.0 & 32.9 & 32.2 \\
\hline Atividade terapêutica & 29.2 & 24.9 & 36.7 & 41.7 & 21.2 & 33.5 \\
\hline Rendimento esportivo & 11.4 & 11.0 & 8.3 & 11.3 & 15.8 & 9.1 \\
\hline
\end{tabular}

Tabela 5

Escala de opinião referente ao nível de importância para permanência nos programas de exercícios físicos, segundo o(s) tipo(s) de exercício(s) físico(s)

\begin{tabular}{lccccc}
\hline \multirow{2}{*}{ Categorias } & \multirow{2}{*}{ Total } & \multicolumn{4}{c}{ Tipos de exercícios físicos } \\
\cline { 5 - 6 } & & Individuais & Coletivos & Ginásticas & Lutas \\
\hline Bem-estar pessoal & 92.0 & 93.7 & 91.4 & 90.2 & 90.0 \\
Prevenção de doenças & 82.8 & 86.1 & 90.0 & 79.8 & 70.0 \\
Condicionamento físico & 80.7 & 80.3 & 85.2 & 80.1 & 80.0 \\
Empatia com o professor & 79.2 & 82.6 & 85.2 & 75.8 & 86.8 \\
Metodologia de trabalho & 75.2 & 78.2 & 82.5 & 71.7 & 85.0 \\
Diversão & 73.9 & 80.4 & 80.5 & 68.2 & 83.3 \\
Ambiente físico & 58.8 & 63.8 & 76.4 & 53.2 & 65.0 \\
Facilidade de horário e acesso ao local da prática & 50.2 & 50.1 & 59.6 & 49.5 & 40.0 \\
Relacionamento social & 49.3 & 52.8 & 68.1 & 44.2 & 46.7 \\
Estética corporal & 48.2 & 46.0 & 46.7 & 49.5 & 90.0 \\
Custo & 47.2 & 47.0 & 54.3 & 46.1 & 80.0 \\
Influência de terceiros & 42.2 & 57.4 & 54.3 & 38.8 & 20.0 \\
Desafio & 40.7 & 43.8 & 46.4 & 37.4 & 65.0 \\
Reconhecimento social & 32.4 & 32.9 & 49.1 & 29.9 & 40.0 \\
Atividade terapêutica & 29.2 & 32.9 & 47.9 & 25.1 & .0 \\
Rendimento esportivo & 11.4 & 12.3 & 33.3 & 8.3 & 30.0 \\
\hline
\end{tabular}


A tabela 5 destaca as semelhanças e as diferenças encontradas para justificar a permanência dos indivíduos, segundo $\mathrm{o}(\mathrm{s})$ tipo(s) de exercício(s) físico(s) praticado(s). Além das categorias que indicam a importância do bem-estar pessoal, da prevenção de doenças, do condicionamento físico, da empatia com o professor, da metodologia de trabalho e da diversão para justificar a permanência nos programas de exercícios físicos, o grupo que pratica esportes coletivos incluiu, como muito importante, os motivos relacionados ao "ambiente físico", com $76.4 \%$ de registros. O grupo de lutas, além das categorias que representam os motivos que justificam a adesão da amostra como um todo, indica também, como determinantes da adesão, motivos relacionados à "estética corporal" (90.0\%) e ao menor "custo" (80.0\%) para praticar a atividade em foco. Por fim, os indivíduos que praticam as modalidades de ginástica não classificaram a categoria "diversão" entre aquelas pontuadas com percentual igual ou maior a $70 \%$.

\section{DISCUSSÃO}

Para melhor discutir os resultados obtidos optou-se por abordá-los em duas sessões. Inicialmente são analisadas as características sócio-demográficas da amostra. Em seguida são discutidos os motivos associados à permanência dos praticantes no programa investigado. Quanto a esta segunda linha de discussão, pode-se afirmar que a mesma trata os motivos de permanência, uma vez que $74.6 \%$ dos participantes apresentam mais de seis meses de prática regular de exercícios físicos, o que é considerando um bom nível de adesão (Dishman, 1998).

De acordo com Dishman (1998), os fatores pessoais, mais especificamente os fatores sociodemográficos, exercem importante influência sobre a adesão de indivíduos nos programas de exercícios físicos. São mais facilmente encontrados entre os indivíduos que aderiram à prática de exercícios físicos, aqueles que dispõem de bom nível econômico e de escolaridade, adultos e casados. Os dados encontrados na presente investigação (tabela 2) confirmam a argumentação do autor, destacando, ainda o sexo feminino e a ginástica (localizada, aeróbica, musculação e/ou hidroginástica), como a categoria de maior preferência dos sujeitos estudados.

Esses resultados demonstram similaridade com outros estudos que afirmam que o status econômico tem relação direta com a adesão à prática de exercícios físicos (Palma et al., 2006; Pate et al., 1995). Na presente investigação os participantes dos programas de exercício físico do SESC-DF com renda familiar acima de R\$ 3.000,00 constituem a maioria da amostra. Nunes e Barros (2004) e Vieira e Ferreira (2004) adicionam ainda uma relação direta entre escolaridade e a prática de exercícios físicos, corroborando os resultados verificados nesta investigação, onde $41.4 \%$ dos indivíduos possuem nível superior (ver tabela 2 ).

Outro aspecto que parece influenciar na adesão à prática de exercícios físicos refere-se ao estado civil dos praticantes. No presente estudo, os indivíduos em situação de união estável representam $51.9 \%$ da amostra, enquanto os solteiros respondem por $34.1 \%$ (tabela 2). Esses dados equivalem-se aos achados de Vieira e Ferreira (2004) e Storchi e Nahas (1992). Para estes autores, a maior procura dos indivíduos com união estável por programas de exercícios físicos em suas investigações está relacionada ao fato de suas amostras serem constituídas, na sua maioria, por sujeitos de meia-idade. Tal interpretação não pode ser totalmente transferida para os dados encontrados na presente investigação, uma vez que a maioria da nossa amostra (44.6\%) apresenta idade compreendida entre 21 e 40 anos, embora somados aos $28.2 \%$ de indivíduos na faixa de 41-60 anos, percebe-se que a maioria é de adultos jovens, portanto, em idade de constituir família (tabela 2).

No que diz respeito à modalidade de exercício físico praticada, foi constatada que a presença masculina é maior em esportes coletivos como o futebol e o vôlei, assim como 
na musculação e na corrida. Entre o grupo feminino, a preferência é pela ginástica, hidroginástica, dança e caminhada (tabela 2). A ginástica aparece como opção de grande procura entre as oferecidas (57.9\%), o que pode ser explicado pelo fato da maior parte dos praticantes de exercícios físicos do SESC-DF ser constituída por mulheres (64.9\%). Este percentual contradiz os dados de outros estudos, que demonstram que os homens são mais fisicamente ativos no lazer do que as mulheres (Monteiro et al., 2003; Salles-Costa, Heilborn, Werneck, Faerstein, \& Lopes, 2003).

Quanto aos motivos associados à permanência dos indivíduos nos programas de exercícios físicos investigados, foi possível verificar pouca variação dos propósitos indicados, quando se comparou o resultado geral (tabela 3) com os parciais obtidos a partir da análise das variáveis idade, sexo e tipos de exercícios físicos (tabelas 4 e 5 , respectivamente). A categoria "bem-estar pessoal" foi considerada como o principal motivo de permanência em todas as variáveis analisadas (idade, sexo e tipos de exercícios físicos). Nota-se que o fato de os indivíduos relatarem positivamente o estado de se sentir bem e saudável, conjuntamente a outros aspectos como diversão, satisfação, qualidade das aulas e prevenção de enfermidades, parecem qualificar os motivos para a permanência nas práticas de exercícios físicos do SESC-DF.

Vale ressaltar a não indicação de propósitos relacionados à estética entre os motivos mais importantes para a maioria dos sujeitos participantes dos programas de exercícios físicos do SESC-DF. Autores como Alves, Pinto, Alves, Mota e Leirós (2009) apontam que é cada vez mais evidente que a insatisfação corporal é uma realidade para ambos os sexos e que o enquadramento corporal nos padrões estéticos estabelecidos culturalmente tem levado os indivíduos à procura de recursos como a prática de exercícios físicos exagerados. Para o grupo investigado nesta investigação, nem mesmo para a faixa etária de 15 a 20 anos esse foi um motivo latente (tabela 4).
Em recente trabalho envolvendo a amostra aqui estudada, Castro et al. (2009) verificaram o aconselhamento médico como fator que tiveram destaque entre os principais motivos que influenciaram o ingresso dos indivíduos nos programas de exercícios físicos. Isso demonstra que predominaram as razões de ordem normativa e utilitária (Lovisolo, 1995). $\mathrm{Na}$ presente investigação, a indicação médica não figurou entre os aspectos mais importantes para justificar a manutenção dos indivíduos na prática de exercícios físicos. Esse resultado sugere que a ação normativa da prescrição médica acerca da prática de exercícios físicos visando à prevenção de doenças ou à recuperação da saúde, parece não figurar de forma preponderante para justificar a permanência dos indivíduos nas práticas de exercícios físicos. A sensação de se sentir bem física e emocionalmente, o reconhecimento da qualidade do trabalho desenvolvido pelo professor, a empatia com o professor, a diversão e o alcance do condicionamento físico são apontados no mesmo grupo de motivos principais, além do objetivo de prevenir doenças.

Dishman (1998) e Andreotti e Okuma (2003) advogam que a prescrição médica e as estratégias centradas nos fatores de risco têm alto potencial para estimular a inserção dos indivíduos em programas de exercícios físicos. No entanto, demonstram baixo valor para reforçar a permanência. Assim, no sentido da manutenção da prática de exercícios físicos, percebe-se, no presente estudo, a combinação de vários fatores que determinam $\mathrm{o}$ comportamento humano. É evidente a combinação de motivos relacionados ao gosto e à utilidade (Lovisolo, 1995), além das características do programa de exercícios físicos (Andreotti \& Okuma, 2003; Dishman, 1998). Segundo Lovisolo (1995), as pessoas tendem a desistir de uma prática utilitária, caso percebam que o custo dessa ação (no sentido amplo: de esforço físico, investimento financeiro, de tempo, etc.) supera seus benefícios. Dessa forma, os resultados da 
presente investigação sugerem que a permanência é justificada pela percepção dos indivíduos de que existe uma boa relação entre o que lhes é útil, atendendo seus objetivos funcionais e o que lhes dá satisfação, percebendo benefícios na perspectiva do prazer e do bem-estar.

Da mesma forma, os dados encontrados reforçam o que preconiza a Teoria SocialCognitiva (Dishman, 1994), em que os componentes específicos sobre características pessoais referentes às habilidades e à autoeficácia, combinados com vários fatores pessoais, ambientais e comportamentais, funcionam em interação e são determinantes entre si. Como exemplo, pode-se destacar a constatação pelos sujeitos de que seus objetivos podem ser alcançados, estimulando a motivação e a manutenção da prática de exercícios físicos. Esse fato corrobora também o Modelo Transteórico dos Estágios de Mudança do Comportamento (Dishman, 1994). Este recomenda que no estágio de manutenção da ação sejam evidenciados os resultados alcançados, de modo a elevar a confiança na capacidade de manter $o$ comportamento desejado e estimular sua continuidade. No estudo desenvolvido por Castro et al. (2009), os indivíduos alegam ingressar nos programas de exercícios físicos motivados pela possibilidade de melhoria do condicionamento físico. Tal interesse também se apresenta nos motivos de permanência na presente investigação. Porém, não somente como desejo, mas na constatação da consecução do seu objetivo percebido, possivelmente, através do aumento da disposição diária.

Monteiro et al. (2003), em estudo sobre a descrição epidemiológica da atividade física no tempo de lazer, entre os anos 1996-1997, perceberam que o principal motivo para a prática de exercícios físicos por parte de 11033 respondentes era a recreação, quando o tipo de atividade era relacionado a esportes coletivos como futebol, voleibol e basquetebol. $\mathrm{Na}$ sequência, foram indicadas a saúde e a estética.
Quando a atividade era de caminhada ou corrida, a recreação foi considerada por $43.1 \%$ dos homens e $41.7 \%$ das mulheres; a saúde obteve $40.7 \%$ entre os homens e $36.8 \%$ entre as mulheres; e a estética $6.0 \%$ e $14.9 \%$, respectivamente. Nas atividades de ginástica, $40.7 \%$ dos homens relataram a recreação como o principal motivo, $19.1 \%$ indicaram a saúde e $22.5 \%$, a estética. Entre as mulheres, $38.3 \%$ indicaram a recreação, $30.9 \%$ a estética e $26.0 \%$ a saúde. $\mathrm{Na}$ mesma pesquisa verificouse que os motivos relacionados à saúde são mais preponderantes quando os exercícios físicos são praticados mais vezes na semana, passando de $4.5 \%$ quando praticados pelos homens em 1 ou 2 dias/semana, para $34.8 \%$ quando praticados em 5 ou mais dias/semana. Para as mulheres essa relação foi de $25.3 \%$ em 1 ou 2 dias/semana, aumentando para $37.7 \%$ em 5 ou mais dias/semana.

Alves et al. (2007) constataram resultados semelhantes aos da presente investigação. Os motivos bem-estar pessoal, condicionamento físico, prevenção de doenças, diversão e empatia com o professor foram os mais destacados, nessa ordem de importância. Esse painel de motivos sugere que os aspectos socioculturais dos exercícios físicos, as características dos programas, o atendimento prestado pelo professor de educação física e a percepção dos benefícios proporcionados e dos objetivos alcançados formam um importante conjunto de justificativas para que os indivíduos permaneçam praticando exercícios físicos.

\section{CONCLUSÕES}

Considerando os objetivos da presente investigação, podemos concluir que o bemestar pessoal foi considerado o principal motivo alegado pelos participantes dos programas de exercícios físicos oferecidos pelo Serviço Social do Comércio, unidades do Distrito Federal - Brasil, entre todas as possibilidades existentes. Em sequência de importância foram justificados a prevenção de doenças, o condicionamento físico, a empatia 
com o professor, a metodologia de trabalho desenvolvida pelo professor e a diversão. Enquanto a prescrição médica tem alto potencial para estimular a inserção destes praticantes nos referidos programas, este motivo perde força no sentido de justificar a permanência da prática de exercícios físicos. Desta forma, ressalta-se a relevância destes achados, uma vez que as políticas públicas de promoção de atividade física e saúde deveriam considerar alguns aspectos que, por vezes, não são contemplados, como a possibilidade de se sentir bem com a prática de atividades físicoesportivas. Contudo, mais investigações desta natureza deveriam ser realizadas, especialmente, com o intuito de detectar as categorias de razões para a não prática de atividades físico-esportivas.

\section{REFERÊNCIAS}

Alves, D., Pinto, M., Alves, S., Mota, A., \& Leirós, V. (2009). Cultura e imagem corporal. Motricidade, $5,1-20$.

Alves, M. P., Junger, W. L., Palma, A., Monteiro, W. D., \& Resende, H. G. (2007). Motivos que justificam a adesão de adolescentes à prática da natação: Qual o espaço ocupado pela saúde? Revista Brasileira de Medicina do Esporte, 13, 421426.

Andreotti, M. C., \& Okuma, S. S. (2003). Perfil sociodemográfico e de adesão inicial de idosos ingressantes em um programa de educação física. Revista Paulista de Educação Física, 17, 142153.

Babbie, E. (2001). Métodos de pesquisa de survey. Belo Horizonte: UFMG.

Bellack, A., Kliebard, H., R., \& Smith, F. (1966). The language of the classroom. New York, Columbia University Press.

Castro, M. S., Miranda, M., Silva, N. L., Palma, A., \& Resende, H. G. (2009). Motivos de ingresso nos programas de exercícios físicos oferecidos pelo Serviço Social do Comércio - DF. Revista Movimento, 15, 87-102.

C.D.C. (2000). Promoting physical activity: A best buy in public health. Atlanta: Autor.

Dishman, R. K. (1994). The measurement conundrum in exercise adherence research. Medicine $\mathcal{E}$ Science in Sports $\&$ Exercise, 26, 1382 1390.
Dishman, R. K. (1998). Exercise adherence: Its impact on public health. Champaign: Human Kinetics.

Fechio, J. J., \& Malerbi, F. E. K. (2004). Adesão a um programa de atividade física em adultos portadores de diabetes. Arquivos Brasileiros de Endocrinologia \& Metabologia, 48, 267-275.

Ferreira, M. S., \& Najar, A. L. (2005). Programas e campanhas de promoção da atividade física. Ciência \& Saúde Coletiva, 10, 207-219.

Hallal, P. C., Bertoldi, A. D., Gonçalves, H., \& Victora, C. G. (2006). Prevalência de sedentarismo e fatores associados em adolescentes de 10-12 anos de idade. Caderno de Saúde Pública, 22, 1277-1287.

Haskell, W. L., Lee, I. M., Pate, R. R., Powell, K. E., Blair, S. N., Franklin, B. A. et al (2007). Physical activity and public health: Updated recommendation for adults from the American College of Sports Medicine and the American Heart Association. Circulation, 28, 1081-1093.

Lovisolo, H. (1995). Normas, utilidades e gostos na aprendizagem. In S. J. Votre \& V. L. M. Costa (Eds.), Cultura, atividade corporal e esporte (pp. 213-231). Rio de Janeiro: Central/UGF.

Lovisolo, H. (2000). Atividade fisica, educação e saúde. Rio de Janeiro: Sprint.

Mira, C. M. (2003). Exercício físico e saúde: Da crítica prudente. In M. Bagrichevsky, A. Palma, \& A. Estevão (Eds.), A saúde em debate na educação física (pp. 169-191). Blumenau: Edibes.

Monteiro, C. A., Conde, W. L., Matsudo, S. M., Matsudo, V. R., Bonseñor, I. M., \& Lotufo, P. A. (2003). A descriptive epidemiology of leisure-time physical activity in Brazil, 19961997. Revista Panamericana de Salud Publica, 14, 246-254.

Nunes, J. O. M., \& Barros, J. F. (2004). Fatores de risco associados à prevalência de sedentarismo em trabalhadores da indústria e da Universidade de Brasília. Lecturas: Educación Física y Deportes, 69(10). Consultado em 17 de Junho de 2005 a partir de http://www.efdeportes.com/efd69/risco.htm

Palma, A., Ferreira, D. C., Bagrichevsky, M., \& Resende, H. G. (2006). Dimensões epidemiológicas associativas entre indicadores sócio-ecônomicos de vida e prática de exercícios físicos. Revista Brasileira de Ciências do Esporte, 27, 119-136.

Pate, R. R., O’Neill, J. R., \& Lobelo, F. (2008). The evolving definition of "sedentary". Exercise and Sport Sciences Reviews, 36, 173-178. 
Pate, R. R., Pratt, M., Blair, S. N., Haskell, W. L., Macera, C. A., Bouchard, C. et al (1995). Physical activity and public health: A recommendation from the Centers for Disease Control and Prevention and the American College of Sports Medicine. The Journal of the American Medical Association, 273, 402-407.

Salles-Costa, R., Heilborn, M. L., Werneck, G. L., Faerstein, E., \& Lopes, C. S. (2003). Gênero e prática de atividade física no lazer. Caderno de Saúde Pública, 19, S325-S333.

Serviço Social do Comércio - Departamento Regional de São Paulo (2003). Escolhas sobre o corpo: Valores e práticas em tempo de mudança. São Paulo: SESC/SP.

Souza, G. S., \& Duarte, M. F. S. (2005). Estágios de mudança de comportamento relacionados à atividade física em adolescentes. Revista Brasileira de Medicina do Esporte, 11,104-108.

Storchi, C. M., \& Nahas, M. V. (1992). A prática espontânea de atividades físicas nas ruas de Florianópolis, SC: Diagnóstico preliminar com indivíduos de meia idade. Revista Brasileira de Ciência e Movimento, 6, 7-13.
U. S. Department of Health and Human Services (1996). Physical activity and health: A report of the surgeon general. Atlanta, GA: US Department of Health and Human Services, Centers for Disease Control and Prevention, National Center for Chronic Disease prevention and Health Promotion.

Varo, J. J., Martínez-González, M. A., Irala-Estévez, J., Kearney, J., Gibney, M., \& Martínez, J. A. (2003). Distribution and determinants of sedentary lifestyles in the European Union. International Journal of Epidemiology, 32, 138-146.

Vieira, V., \& Ferreira, M. S. (2004). Perfil de praticantes de atividade física na pista do Maracanã. Revista Ação e Movimento, 1, 81-90.

Waxman, A. (2004). WHO's global strategy on diet, physical activity and health: Response to a worldwide epidemic of non-communicable diseases. Scandinavian Journal of Nutrition, 48(2), 58-60.

Williams, P. T. (2003). The illusion of improved physical fitness and reduced mortality. Medicine $\mathcal{E}$ Science in Sports \& Exercise, 35, 736-740. 